\title{
Variation Inequalities for the Commutators of One-Sided Calderón-Zygmund Singular Integrals with Lipschitz Functions
}

\author{
Tingting Juan, Xianming Hou* \\ School of Mathematics and Statistics, Linyi University, Linyi, China \\ Email: jtt13969823573@163.com, ^houxianming37@163.com
}

How to cite this paper: Juan, T.T. and Hou, X.M. (2021) Variation Inequalities for the Commutators of One-Sided Calderón-Zygmund Singular Integrals with Lipschitz Functions. Advances in Pure Mathematics, 11, 978-987.

https://doi.org/10.4236/apm.2021.1112063

Received: November 22, 2021

Accepted: December 27, 2021

Published: December 30, 2021

Copyright $\odot 2021$ by author(s) and Scientific Research Publishing Inc. This work is licensed under the Creative Commons Attribution International License (CC BY 4.0).

http://creativecommons.org/licenses/by/4.0/

\begin{abstract}
In this paper, we establish the weighted $L^{p}(1<p<\infty)$ boundedness of variation operator for the commutators generated by one-sided Calderón-Zygmund singular integrals with Lipschitz functions.
\end{abstract}

\section{Keywords}

Variation, One-Sided Singular Integrals, Commutator, Lipschitz Functions

\section{Introduction}

Let $\mathcal{T}=\left\{T_{\varepsilon}\right\}_{\varepsilon}$ be a family of operators such that the limit $\lim _{\varepsilon \rightarrow 0} T_{\varepsilon} f$ exists. It is nature to study the speed of convergence of the family $\left\{T_{\varepsilon}\right\}$. A classic method is to consider square function of the type $\left(\sum_{i=1}^{\infty}\left|T_{\varepsilon_{i}} f-T_{\varepsilon_{i}+1} f\right|^{2}\right)^{1 / 2}$, more generally, define the following oscillation operator:

$$
\mathcal{O}(\mathcal{I f})(x)=\left(\sum_{i=1}^{\infty} \sup _{t_{i+1} \leq \varepsilon_{i+1}<\varepsilon_{i} \leq t_{i}}\left|T_{\varepsilon_{i}+1} f(x)-T_{\varepsilon_{i}} f(x)\right|^{2}\right)^{1 / 2},
$$

where $\left\{t_{i}\right\}$ is a fixed sequence decreasing to zero. Let $\rho>2$. The $\rho$-variation operator is defined by

$$
\mathcal{V}_{\rho}(\mathcal{I f})(x)=\sup _{\varepsilon_{i} \backslash 0}\left(\sum_{i=1}^{\infty}\left|T_{\varepsilon_{i}+1} f(x)-T_{\varepsilon_{i}} f(x)\right|^{\rho}\right)^{1 / \rho},
$$

where the sup is taken over all sequence $\left\{\varepsilon_{i}\right\}$ of positive numbers decreasing to zero. The variation inequalities play important roles in probability, ergodic theory, and harmonic analysis. We refer the readers to [1]-[6] and the references therein for more background information. Gillespie and Torrea [7] show that 
oscillation and variation of Hilbert transforms are bounded on $L^{p}(\omega)$ for $1<p<\infty$. The weighted oscillation and variation boundedness of differential operators and Calderón-Zygmund singular integral are established in [8] [9]. This paper is devoted to studying weighted boundedness of $\rho$-variational operator for the families of commutator generated by one-sided Calderón-Zygmund singular integral with Lipschitz functions. Recently, Liu and $\mathrm{Wu}$ [10] presented a criterion on the weighted estimate of the oscillation and variation operators for the commutators of Calderón-Zygmund singular integrals with BMO functions in one dimension. Variation inequalities for the commutators one-sided singular integrals with BMO functions were established in [11]. Zhang and $\mathrm{Wu}$ [12] gave the oscillation and variation inequalities for the commutators of singular integrals with Lipschitz functions.

Before stating our main results, we firstly recall some notations and definitions. In [13], Aimar, Forzani and Martín-Reyes introduced the one-sided Calderón-Zygmund singular integrals defined by:

$$
T^{+} f(x)=\lim _{\varepsilon \rightarrow 0^{+}} T_{\varepsilon}^{+} f(x)=\lim _{\varepsilon \rightarrow 0^{+}} \int_{x+\varepsilon}^{\infty} K(x-y) f(y) \mathrm{d} y
$$

and

$$
T^{-} f(x)=\lim _{\varepsilon \rightarrow 0^{+}} T_{\varepsilon}^{-} f(x)=\lim _{\varepsilon \rightarrow 0^{+}} \int_{-\infty}^{x-\varepsilon} K(x-y) f(y) \mathrm{d} y,
$$

where the kernel $K$ is called the one-sided Calderón-Zygmund kernel (OCZK) which satisfies

$$
\begin{gathered}
\left|\int_{a<|x|<b} K(x) \mathrm{d} x\right| \leq C, \quad 0<a<b, \\
|K(x)| \leq C /|x|, \quad x \neq 0, \\
|K(x-y)-K(x)| \leq C|y| /|x|^{2}, \quad|x|>2|y|>0,
\end{gathered}
$$

with support in $\mathbb{R}^{-}=(-\infty, 0)$ or $\mathbb{R}^{+}=(0,+\infty)$, where (1.4) is named Hörmander's condition. Equation (1.3) is also called the size condition for $K$. An interesting example is

$$
K(x)=\frac{\sin (\log |x|)}{x \log |x|} \chi_{(-\infty, 0)}(x),
$$

where $\chi_{E}$ denotes the characteristic function of a set $E$, for more details one can refer to [13].

For $0<\alpha<1$, a function $f \in \operatorname{Lip}_{\alpha}$, if it satisfies

$$
\|f\|_{L i_{\alpha}}=\sup _{x, h \in \mathbb{R}, h \neq 0} \frac{f(x+h)-f(x)}{|h|^{\alpha}}<\infty .
$$

Let $K$ be one-sided Calderón-Zygmund kernel (OCZK) with support in $\mathbb{R}^{-}=(-\infty, 0) . \quad b \in \operatorname{Lip}_{\alpha}$, we define the following one-sided operator

$$
T_{b}^{+} f(x)=\lim _{\varepsilon \rightarrow 0^{+}} T_{b, \varepsilon}^{+} f(x),
$$

where 


$$
T_{b, \varepsilon}^{+} f(x)=\int_{x+\varepsilon}^{\infty}(b(x)-b(y)) K(x-y) f(y) \mathrm{d} y .
$$

In this paper, we study variational inequalities for the commutators of one-sided singular integrals with Lipschitz functions. Our result can be formulated as follows:

Theorem 1.1. Let $K$ be one-sided Calderón-Zygmund kernel (OCZK) with support in $\mathbb{R}^{-}=(-\infty, 0)$. Let $\mathcal{T}_{b}=\left\{T_{b, \varepsilon}^{+}\right\}_{\varepsilon>0}$ and $\mathcal{T}=\left\{T_{\varepsilon}^{+}\right\}_{\varepsilon>0}$ be given as in (1.5) and (1.1), respectively. The operator $\mathcal{V}_{\rho}\left(\mathcal{T}^{+}\right)$is bounded in $L^{p_{0}}(\mathbb{R}, d x)$ for some $p_{0} \in(1, \infty)$. Let $0<\alpha<1, b \in$ Lip $_{\alpha}$. Then, for all $\omega \in A^{+}(p, q)$, $1<p<q<\infty, 1 / p-1 / q=\alpha$, we have

$$
\left\|\mathcal{V}_{\rho}\left(\mathcal{T}_{b}^{+}\right) f\right\|_{L^{q}\left(w^{q}\right)} \lesssim\|b\|_{L i_{a}}\|f\|_{L^{p}\left(w^{p}\right)}
$$

The rest of this paper is organized as follows. In Section 2, we introduce and recall some basic facts and auxiliary lemmas. The proof of main theorem will be given in Sections 3.

Throughout this paper, the letter $C$, sometimes with additional parameters, will stand for positive constants, not necessarily the same one at each occurrence, but independent of the essential variables. We also denote $f \lesssim g$ if $f \leq C g$.

\section{Preliminaries}

In 1986, Sawyer [14] first introduced the one-sided Muckenhoupt weights $A_{p}^{+}$ and $A_{p}^{-}$to treat the one-sided Hardy-Littlewood maximal operators

$$
M^{+} f(x):=\sup _{h>0} \frac{1}{h} \int_{x}^{x+h}|f(y)| \mathrm{d} y, \quad M^{-} f(x):=\sup _{h>0} \frac{1}{h} \int_{x-h}^{x}|f(y)| \mathrm{d} y .
$$

A positive function $\omega$ is said to belong to $A_{p}^{+}$or $A_{p}^{-}$if it satisfies

$$
A_{p}^{+}(\omega):=\sup _{a<b<c} \frac{1}{(c-a)^{p}} \int_{a}^{b} \omega(x) \mathrm{d} x\left(\int_{b}^{c} \omega(x)^{1-p^{\prime}} \mathrm{d} x\right)^{p-1}<\infty
$$

or

$$
A_{p}^{-}(\omega):=\sup _{a<b<c} \frac{1}{(c-a)^{p}} \int_{b}^{c} \omega(x) \mathrm{d} x\left(\int_{a}^{b} \omega(x)^{1-p^{\prime}} \mathrm{d} x\right)^{p-1}<\infty,
$$

when $1<p<\infty$; also, for $p=1$,

$$
A_{1}^{+}: M^{-} \omega \leq C \omega, A_{1}^{-}: M^{+} \omega \leq C \omega,
$$

for some constant $C$. If $1 \leq p<\infty$, then $A_{p} \varsubsetneqq A_{p}^{+}$and $A_{p} \varsubsetneqq A_{p}^{-}$. Notice that the function $\omega(x)=\mathrm{e}^{x}$ mentioned above is in $A_{p}^{+}$but not in $A_{p}$.

Similarly, the double weight classes $A^{+}(p, q)$ and $A^{-}(p, q)$ are denoted by

$$
\begin{aligned}
& A^{+}(p, q): \frac{1}{(c-a)^{1-\alpha}}\left(\int_{a}^{b} \omega^{q}\right)^{1 / q}\left(\int_{b}^{c} \omega^{-p^{\prime}}\right)^{1 / p^{\prime}}<C, \\
& A^{-}(p, q): \frac{1}{(c-a)^{1-\alpha}}\left(\int_{b}^{c} \omega^{q}\right)^{1 / q}\left(\int_{a}^{b} \omega^{-p^{\prime}}\right)^{1 / p^{\prime}}<C
\end{aligned}
$$

for all $a<b<c \in \mathbb{R}, 0<\alpha<1,1<p<q$ and $1 / p-1 / q=\alpha$; also for $p=1$, 
$1-1 / q=\alpha$,

$$
A(1, q)^{+}: M^{-} \omega^{q} \lesssim \omega^{q}, A(1, q)^{-}: M^{+} \omega^{q} \lesssim \omega^{q} .
$$

The one-sided $A_{p}^{+}$classes not only control the boundedness of one-sided Hardy-Littlewood maximal operators, but also serve as the right weight classes for one-sided singular integral operators. Set $1<p<\infty$ and let $K$ be an OCZK with support in $\mathbb{R}^{-}$. Then $T^{+}$is bounded on $L^{p}(\omega)$ if $\omega \in A_{p}^{+}$, see [13].

Lemma 2.1. [15] Suppose that $\omega \in A_{1}^{-}$, then there exists $\varepsilon_{1}>0$ such that, for all $1<r \leq 1+\varepsilon_{1}, \omega^{r} \in A_{1}^{-}$.

Lemma 2.2. [16] Suppose $\omega \in A^{+}(p, q)$, then $\omega^{q} \in A_{q}^{+}$and $\omega^{p} \in A_{p}^{+}$for all $1<p<q \leq \infty$.

Lemma 2.3. [17] Let $1<p_{0}<\infty$ and let $T$ be sublinear operator defined in $C_{c}^{\infty}(\mathbb{R})$ satisfying

$$
\|\omega T f\|_{\infty} \leq C\|f \omega\|_{p_{0}}
$$

for every $x \in \mathbb{R}$ and $\omega \in A^{+}\left(p_{0}, \infty\right)$; then, for every $1<p<p_{0}$, $1 / p-1 / q=1 / p_{0}$, and $\omega \in A^{+}(p, q)$, the inequality

$$
\|\omega T f\|_{q} \leq C\|f \omega\|_{p}
$$

Lemma 2.4. [18] For every $p$ with $p_{0} \leq p<\infty$, assume that $\omega \in A_{\infty}^{+}$, and that $M^{+} f \in L^{p_{0}}(\omega)$ for some $p_{0}$ with $0<p_{0}<\infty$. Then

$$
\left\|M^{+} f\right\|_{L^{p}(\omega)} \leq C\left\|M^{+, \#} f\right\|_{L^{p}(\omega)},
$$

where

$$
M^{+, \#} f(x)=\sup _{h>0} \frac{1}{h} \int_{x}^{x+h}\left(f(y)-\frac{1}{h} \int_{x+h}^{x+2 h} f(z) \mathrm{d} z\right)^{+} \mathrm{d} y
$$

and $z^{+}=\max \{z, 0\}$.

\section{The Proof of Theorem 1.1}

According to [7], we denote by $F_{\rho}$ the mixed norm Banach space of two variable functions $h$ defined on $\mathbb{N} \times \Theta$ such that

$$
\|h\|_{F_{\rho}}=\sup _{\beta}\left(\sum_{i}|h(i, \beta)|^{\rho}\right)^{1 / \rho}<\infty
$$

where $\Theta=\left\{\beta: \beta=\left\{\varepsilon_{i}\right\}, \varepsilon_{i} \in \mathbb{R}, \varepsilon_{i} \searrow 0\right\}$. Given a family of operators $\mathcal{T}=\left\{T_{t}^{+}\right\}_{t>0}$ defined on $L^{p}(\mathbb{R})$, we consider the $F_{\rho}$-valued operator $V(\mathcal{T}): f \rightarrow V(\mathcal{T}) f$ on $L^{p}(\mathbb{R})$ defined by

$$
V(\mathcal{T}) f(x)=\left\{T_{\varepsilon_{i}+1} f(x)-T_{\varepsilon_{i}} f(x)\right\}_{\beta=\left\{\varepsilon_{i}\right\} \in \Theta}=:\left\{T_{\left[\varepsilon_{i}+1, \varepsilon_{i}\right]} f(x)\right\}_{\beta=\left\{\varepsilon_{i}\right\} \in \Theta},
$$

where $\left\{T_{\left[\varepsilon_{i}+1, \varepsilon_{i}\right]} f(x)\right\}_{\beta=\left\{\varepsilon_{i}\right\} \in \Theta}$ is an abbreviation for the element of $F_{\rho}$ given by

$$
(i, \beta)=\left(i,\left\{\varepsilon_{i}\right\}\right) \rightarrow T_{\left[\varepsilon_{i}+1, \varepsilon_{i}\right]} f(x) .
$$

This implies 


$$
\mathcal{V}_{\rho}(\mathcal{T} f)(x)=\|V(\mathcal{T}) f(x)\|_{F_{\rho}} .
$$

This section is devoted to proving Theorem 1.1. To do this, we need establish the following lemma.

Lemma 3.1. Let $K$ be one-sided Calderón-Zygmund kernel (OCZK) with support in $\mathbb{R}^{-}=(-\infty, 0)$. Let $\mathcal{T}_{b}=\left\{T_{b, \varepsilon}^{+}\right\}_{\varepsilon>0}$ and $\mathcal{T}=\left\{T_{\varepsilon}^{+}\right\}_{\varepsilon>0}$ be given as in (1.5) and (1.1), respectively. The operator $\mathcal{V}_{\rho}\left(\mathcal{T}^{+}\right)$is bounded in $L^{p_{0}}(\mathbb{R}, d x)$ for some $p_{0} \in(1, \infty)$. Let $0<\alpha<1, b \in$ Lip $_{\alpha}$. Then, for all $\omega \in A^{+}(p, q)$, $1<p<q<\infty, 1 / p-1 / q=\alpha$, we have

$$
\left\|M^{+, \#}\left(\mathcal{V}_{\rho}\left(\mathcal{T}_{b}^{+}\right) f\right)(x)\right\|_{L^{q}\left(w^{q}\right)} \lesssim\|b\|_{L i p_{a}}\|f\|_{L^{p}\left(w^{p}\right)} .
$$

Proof. Let $x \in \mathbb{R}, h>0$ and $I=[x, x+4 h]$. Suppose $f=f_{1}+f_{2}:=f \chi_{I}+f \chi_{I^{c}}$. Notice that

$$
M^{+, \#}\left(\mathcal{V}_{\rho}\left(\mathcal{T}_{b}^{+}\right) f\right)(x) \lesssim \frac{1}{h} \int_{x}^{x+2 h}\left|\mathcal{V}_{\rho}\left(\mathcal{T}_{b}^{+}\right) f(y)-\mathcal{V}_{\rho}(\mathcal{T})\left(\left(b-b_{I}\right) f_{2}\right)(x)\right| \mathrm{d} y
$$

It is easy to check

$$
\begin{aligned}
& \frac{1}{h} \int_{x}^{x+2 h}\left|\mathcal{V}_{\rho}\left(\mathcal{T}_{b}^{+}\right) f(y)-\mathcal{V}_{\rho}(\mathcal{T})\left(\left(b-b_{I}\right) f_{2}\right)(x)\right| \mathrm{d} y \\
& \leq \frac{1}{h} \int_{x}^{x+2 h}\left\|V\left(\mathcal{T}_{b}^{+}\right) f(y)\right\|_{F_{\rho}}-\left\|V\left(\mathcal{T}_{b}^{+}\right)\left(\left(b-b_{I}\right) f_{2}\right)(x)\right\|_{F_{\rho}} \mid \mathrm{d} y \\
& \leq \frac{1}{h} \int_{x}^{x+2 h}\left\|V\left(\mathcal{T}_{b}^{+}\right) f(y)-V\left(\mathcal{T}_{b}^{+}\right)\left(\left(b-b_{I}\right) f_{2}\right)(x)\right\|_{F_{\rho}} \mathrm{d} y \\
& \leq \frac{1}{h} \int_{x}^{x+2 h}\left|b(y)-b_{I}\right| \mathcal{V}_{\rho}\left(\mathcal{T}^{+}\right) f(y) \mathrm{d} y+\frac{1}{h} \int_{x}^{x+2 h} \mathcal{V}_{\rho}\left(\mathcal{T}^{+}\right)\left(\left(b-b_{I}\right) f_{1}\right)(y) \mathrm{d} y \\
& \quad+\frac{1}{h} \int_{x}^{x+2 h}\left\|V\left(\mathcal{T}^{+}\right)\left(\left(b-b_{I}\right) f_{2}\right)(y)-V\left(\mathcal{T}^{+}\right)\left(\left(b-b_{I}\right) f_{2}\right)(x)\right\|_{F_{\rho}} \mathrm{d} y .
\end{aligned}
$$

Consider the following three sublinear operators defined on $C_{c}^{\infty}(\mathbb{R})$ :

$$
\begin{aligned}
& M_{1}^{+} f(x):=\sup _{h>0} \frac{1}{h} \int_{x}^{x+2 h}\left|b(y)-b_{I}\right| \mathcal{V}_{\rho}\left(\mathcal{T}^{+}\right) f(y) \mathrm{d} y, \\
& M_{2}^{+} f(x):=\sup _{h>0} \frac{1}{h} \int_{x}^{x+2 h} \mathcal{V}_{\rho}\left(\mathcal{T}^{+}\right)\left(\left(b-b_{I}\right) f_{1}\right)(y) \mathrm{d} y, \\
& M_{3}^{+} f(x):=\sup _{h>0} \frac{1}{h} \int_{x}^{x+2 h}\left\|V\left(\mathcal{T}^{+}\right)\left(\left(b-b_{I}\right) f_{2}\right)(y)-V\left(\mathcal{T}^{+}\right)\left(\left(b-b_{I}\right) f_{2}\right)(x)\right\|_{F_{\rho}} \mathrm{d} y . \\
& \text { For } M_{1}^{+} f \text {, let } \omega \in A^{+}(1 / \alpha, \infty) \text {, then } \omega^{-1 /(1-\alpha)} \in A_{1}^{-} \text {. By Lemma } 2.1 \text {, there ex- } \\
& \text { ists } t>1 \text { such that } \omega^{-t /(1-\alpha)} \in A_{1}^{-} \text {. Take } r=t /(1-\alpha) \text { and } 1 / s-1 / r=\alpha \text {. Using } \\
& \text { Hölder's inequality and the } L^{r} \text { boundedness for } \mathcal{V}_{\rho}\left(\mathcal{T}^{+}\right), \text {we obtain } \\
& \frac{1}{h} \int_{x}^{x+2 h}\left|b(y)-b_{I}\right| \mathcal{V}_{\rho}\left(\mathcal{T}^{+}\right) f(y) \mathrm{d} y \lesssim \sup _{y \in(x, x+2 h)}\left|b(y)-b_{I}\right|\left(\frac{1}{h} \int_{x}^{x+2 h}|f(y)|^{r} \mathrm{~d} y\right)^{1 / r} \\
& \lesssim\|b\|_{L i p_{\alpha}} h^{\alpha}\left(\frac{1}{h} \int_{x}^{x+2 h}|f(y)|^{r} \omega(y)^{r} \omega(y)^{-r} \mathrm{~d} y\right)^{1 / r} \\
& \lesssim\|b\|_{L i p_{\alpha}}\left(\int_{x}^{x+2 h}|f(y) \omega(y)|^{1 / \alpha} \mathrm{d} y\right)^{\alpha}\left(\frac{1}{h} \int_{x}^{x+2 h} \omega(y)^{-s} \mathrm{~d} y\right)^{1 / s} \\
& \lesssim\|b\|_{L i p_{\alpha}}\|f \omega\|_{L^{1 / \alpha}} \omega(x)^{-1} .
\end{aligned}
$$


By Lemma 2.3, we have that for $1 / r-1 / s=\alpha$ and $A^{+}(p, q)$

$$
\left\|M_{1}^{+} f\right\|_{L^{q}\left(\omega^{q}\right)} \lesssim\|b\|_{L i p_{\alpha}}\|f\|_{L^{p}\left(\omega^{p}\right)} \cdot
$$

For $M_{2}^{+} f$, by Hölder's inequality and the $L^{r}$ boundedness for $\mathcal{V}_{\rho}\left(\mathcal{T}^{+}\right)$, we get

$$
\begin{aligned}
& \frac{1}{h} \int_{x}^{x+2 h} \mathcal{V}_{\rho}\left(\mathcal{T}^{+}\right)\left(\left(b-b_{I}\right) f_{1}\right)(y) \mathrm{d} y \\
& \leq\left(\frac{1}{h} \int_{x}^{x+2 h}\left|\mathcal{V}_{\rho}\left(\mathcal{T}^{+}\right)\left(\left(b-b_{I}\right) f_{1}\right)(y)\right|^{r} \mathrm{~d} y\right)^{1 / r} \\
& \lesssim\left(\frac{1}{h} \int_{x}^{x+2 h}\left|\left(b(y)-b_{I}\right) f_{1}(y)\right|^{r} \mathrm{~d} y\right)^{1 / r} \\
& \leq \sup _{y \in(x, x+2 h)}\left|b(y)-b_{I}\right|\left(\frac{1}{h} \int_{x}^{x+2 h}|f(y)|^{r} \mathrm{~d} y\right)^{1 / r} \\
& \lesssim\|b\|_{L_{\text {ip }}}\|f \omega\|_{L^{1 / \alpha}} \omega(x)^{-1},
\end{aligned}
$$

where $\omega^{-r}=\omega^{-t /(1-\alpha)} \in A_{1}^{-}$for all $\omega \in A^{+}(1 / \alpha, \infty)$. Then

$$
\left\|\omega M_{2}^{+} f\right\|_{\infty} \lesssim\|b\|_{L i p_{\alpha}}\|f \omega\|_{L^{1 / \alpha}} .
$$

For $1 / r-1 / s=\alpha$ and $A^{+}(p, q)$, it follows from Lemma 2.3 that

$$
\left\|M_{2}^{+} f\right\|_{L^{q}\left(\omega^{q}\right)} \lesssim\|b\|_{L i p_{\alpha}}\|f\|_{L^{p}\left(\omega^{p}\right)} \cdot
$$

It remains to deal with $M_{3}^{+} f$. For $y \in[x, x+2 h]$, we get

$$
\begin{aligned}
& T_{\left[\varepsilon_{i+1}, \varepsilon_{i}\right]}^{+}\left(\left(b-b_{I}\right) f_{2}\right)(y)-T_{\left[\varepsilon_{i+1}, \varepsilon_{i}\right]}^{+}\left(\left(b-b_{I}\right) f_{2}\right)(x) \\
& =\int_{\mathbb{R}} k(y-z) \chi_{\left(y+\varepsilon_{i+1}, y+\varepsilon_{i}\right)}(z)\left(b(z)-b_{I}\right) f_{2}(z) \mathrm{d} z \\
& \quad-\int_{\mathbb{R}} k(x-z) \chi_{\left(x+\varepsilon_{i+1}, x+\varepsilon_{i}\right)}(z)\left(b(z)-b_{I}\right) f_{2}(z) \mathrm{d} z \\
& =\int_{\mathbb{R}}(k(y-z)-k(x-z)) \chi_{\left(y+\varepsilon_{i+1}, y+\varepsilon_{i}\right)}(z)\left(b(z)-b_{I}\right) f_{2}(z) \mathrm{d} z \\
& \quad+\int_{\mathbb{R}} k(x-z)\left(\chi_{\left(y+\varepsilon_{i+1}, y+\varepsilon_{i}\right)}(z)-\chi_{\left(x+\varepsilon_{i+1}, x+\varepsilon_{i}\right)}(z)\right)\left(b(z)-b_{I}\right) f_{2}(z) \mathrm{d} z
\end{aligned}
$$

In view of (3.2), we have

$$
\begin{aligned}
& \left\|V\left(\mathcal{T}^{+}\right)\left(\left(b-b_{I}\right) f_{2}\right)(y)-V\left(\mathcal{T}^{+}\right)\left(\left(b-b_{I}\right) f_{2}\right)(x)\right\|_{F_{\rho}} \\
& \leq\left\|\left\{\int_{\mathbb{R}}(k(y-z)-k(x-z)) \chi_{\left(y+\varepsilon_{i+1}, y+\varepsilon_{i}\right)}(z)\left(b(z)-b_{I}\right) f_{2}(z) \mathrm{d} z\right\}_{i \in \mathbb{N}, \beta=\left\{\varepsilon_{i}\right\} \in \Theta}\right\|_{F_{\rho}} \\
& \quad+\|\left\{\int_{\mathbb{R}} k(x-z)\left(\chi_{\left(y+\varepsilon_{i+1}, y+\varepsilon_{i}\right)}(z)-\chi_{\left(x+\varepsilon_{i+1}, x+\varepsilon_{i}\right)}(z)\right)\right. \\
& \left.\quad \times\left(b(z)-b_{I}\right) f_{2}(z) \mathrm{d} z\right\}_{i \in \mathbb{N}, \beta=\left\{\varepsilon_{i}\right\} \in \Theta} \|_{F_{\rho}} \\
& =: J_{1}+J_{2} .
\end{aligned}
$$

Since $z \in(x+8 h, \infty)$ and $y \in(x, x+2 h)$, we get $|x-z| \geq 2|x-y|$. Using (1.4), we have $|k(y-z)-k(x-z)| \lesssim h|x-z|^{-2}$. Note that

$$
\left\|\left\{\chi_{\left(y+\varepsilon_{i+1}, y+\varepsilon_{i}\right]}(z)\right\}_{i \in \mathbb{N}, \gamma=\left\{\varepsilon_{i}\right\} \in \Theta}\right\|_{F_{\rho}} \leq 1 \text {, for } \forall y \in \mathbb{R} \text {. }
$$


Then

$$
\begin{aligned}
J_{1} & \lesssim \int_{\mathbb{R}} \frac{h}{|x-z|^{2}} \mid\left\{\left\{\chi_{\left(y+\varepsilon_{i+1}, y+\varepsilon_{i}\right]}(z)\right\}_{i \in \mathbb{N}, \gamma=\left\{\varepsilon_{i}\right\} \in \Theta} \|_{F_{\rho}}\left|\left(b(z)-b_{I}\right) f_{2}(z)\right| \mathrm{d} z\right. \\
& \lesssim h \int_{\mathbb{R}} \frac{\left|\left(b(z)-b_{I}\right) f_{2}(z)\right|}{|x-z|^{2}} \mathrm{~d} z \\
& \lesssim h \sum_{k=3}^{\infty} \int_{x+2^{k} h}^{x+2^{k+1} h} \frac{\left|\left(b(z)-b_{I}\right) f(z)\right|}{|x-z|^{2}} \mathrm{~d} z \\
& \lesssim\|b\|_{L i p_{\alpha}} \sum_{k=3}^{\infty} \frac{h^{\alpha}}{2^{k(1-\alpha)}} \frac{1}{2^{k+1} h} \int_{x}^{x+2^{k+1} h}|f(z)| \mathrm{d} z \\
& \lesssim\|b\|_{L i p_{\alpha}} \sum_{k=3}^{\infty} \frac{h^{\alpha}}{2^{k(1-\alpha)}}\left(\frac{1}{2^{k+1} h} \int_{x}^{x+2^{k+1} h}|f(y)|^{r} \omega(y)^{r} \omega(y)^{-r} \mathrm{~d} y\right)^{1 / r} \\
& \lesssim\|b\|_{L i p_{\alpha}} \sum_{k=3}^{\infty} \frac{1}{2^{k}}\left(\int_{x}^{x+2^{k+1} h}|f(y) \omega(y)|^{1 / \alpha} \mathrm{d} y\right)^{\alpha}\left(\frac{1}{2^{k+1} h} \int_{x}^{x+2^{k+1} h} \omega(y)^{-s} \mathrm{~d} y\right)^{1 / s} \\
& \lesssim\|b\|_{L i p_{\alpha}}\|f \omega\|_{L^{1 / \alpha}} \omega(x)^{-1},
\end{aligned}
$$

where $\omega^{-r}=\omega^{-t /(1-\alpha)} \in A_{1}^{-}$for all $\omega \in A^{+}(1 / \alpha, \infty)$.

For $\left\{\varepsilon_{i}\right\} \in \Theta$, let $N_{1}=\left\{i \in Z: \varepsilon_{i}-\varepsilon_{i+1} \geq y-x\right\}$ and $N_{2}=\left\{i \in Z: \varepsilon_{i}-\varepsilon_{i+1}<y-x\right\}$. Then

$$
\begin{aligned}
J_{2} \leq & \|\left\{\int_{\mathbb{R}}\left(\chi_{\left(y+\varepsilon_{i+1}, y+\varepsilon_{i}\right)}(z)-\chi_{\left(x+\varepsilon_{i+1}, x+\varepsilon_{i}\right)}(z)\right)\right. \\
& \left.\times k(x-z)\left(b(z)-b_{I}\right) f_{2}(z) \mathrm{d} z\right\}_{i \in \mathbb{N}_{1}, \beta=\left\{\varepsilon_{i}\right\} \in \Theta} \|_{F_{\rho}} \\
\leq & \|\left\{\int_{\mathbb{R}}\left(\chi_{\left(y+\varepsilon_{i+1}, y+\varepsilon_{i}\right)}(z)-\chi_{\left(x+\varepsilon_{i+1}, x+\varepsilon_{i}\right)}(z)\right)\right. \\
& \left.\times k(x-z)\left(b(z)-b_{I}\right) f_{2}(z) \mathrm{d} z\right\}_{i \in \mathbb{N}_{2}, \beta=\left\{\varepsilon_{i}\right\} \in \Theta} \|_{F_{\rho}} \\
=: & J_{21}+J_{22} .
\end{aligned}
$$

Now we estimate $J_{21}$. It is easy to see

$$
\begin{aligned}
J_{21} \leq & \left\|\left\{\int_{\mathbb{R}} k(x-z) \chi_{\left(x+\varepsilon_{i+1}, y+\varepsilon_{i+1}\right)}(z)\left(b(z)-b_{I}\right) f_{2}(z) \mathrm{d} z\right\}_{i \in \mathbb{N}_{1}, \beta=\left\{\varepsilon_{i}\right\} \in \Theta}\right\|_{F_{\rho}} \\
& +\left\|\left\{\int_{\mathbb{R}} k(x-z) \chi_{\left(x+\varepsilon_{i}, y+\varepsilon_{i}\right)}(z)\left(b(z)-b_{I}\right) f_{2}(z) \mathrm{d} z\right\}_{i \in \mathbb{N}_{1}, \beta=\left\{\varepsilon_{i}\right\} \in \Theta}\right\|_{F_{\rho}} \\
= & : L_{1}+L_{2} .
\end{aligned}
$$

For $i \in N_{1}$, using Hölder's inequality and (1.3), we have

$$
\begin{aligned}
L_{1} & \lesssim h^{1 / r^{\prime}}\left\|\left\{\left(\int_{\mathbb{R}} \frac{\left|b(z)-b_{I}\right|^{r}\left|f_{2}(z)\right|^{r}}{|x-z|^{r}} \chi_{\left(y+\varepsilon_{i+1}, y+\varepsilon_{i}\right)}(z) \mathrm{d} z\right)^{1 / r}\right\}_{i \in \mathbb{N}_{1}, \beta=\left\{\varepsilon_{i}\right\} \in \Theta}\right\|_{F_{\rho}} \\
& =h^{1 / r^{\prime}}\left(\sup _{\beta} \sum_{i \in \mathbb{N}_{1}}\left(\int_{\mathbb{R}} \chi_{\left(x+\varepsilon_{i+1}, x+\varepsilon_{i}\right)}(z) \frac{\left|b(z)-b_{I}\right|^{r}\left|f_{2}(z)\right|^{r}}{|x-z|^{r}} \mathrm{~d} z\right)^{p / r}\right)^{1 / \rho}
\end{aligned}
$$




$$
\begin{aligned}
& \lesssim h^{1 / r^{\prime}}\left(\int_{\mathbb{R}} \frac{\left|b(z)-b_{I}\right|^{r}\left|f_{2}(z)\right|^{r}}{|x-z|^{r}} \mathrm{~d} z\right)^{1 / r} \\
& \lesssim\|b\|_{L i p_{\alpha}} \sum_{k=3}^{\infty} \frac{h^{\alpha}}{2^{k\left(1 / r^{\prime}-\alpha\right)}}\left(\frac{1}{2^{k+1} h} \int_{x}^{x+2^{k+1} h}|f(z)|^{r} \mathrm{~d} z\right)^{1 / r} \\
& \lesssim \sum_{k=3}^{\infty} \frac{\|b\|_{L i p_{\alpha}}}{2^{k / r^{\prime}}}\left(\int_{x}^{x+2^{k+1} h} \mid f(z) \omega(z)^{1 / \alpha} \mathrm{d} z\right)^{\alpha}\left(\frac{1}{2^{k+1} h} \int_{x}^{x+2^{k+1} h} \omega(z)^{-s} \mathrm{~d} z\right)^{1 / s} \\
& \lesssim\|b\|_{L i p_{\alpha}}\|f \omega\|_{L^{1 / \alpha}} \omega(x)^{-1} \text {, }
\end{aligned}
$$

where $\omega^{-r}=\omega^{-t /(1-\alpha)} \in A_{1}^{-}$for all $\omega \in A^{+}(1 / \alpha, \infty)$. By a similar estimate of $L_{1}$, we have

$$
L_{2} \lesssim\|b\|_{L i p_{\alpha}}\|f \omega\|_{L^{1 / \alpha}} \omega(x)^{-1} .
$$

Now we estimate $J_{22}$. It is easy to see

$$
\begin{aligned}
J_{22} & \leq\left\|\left\{\int_{\mathbb{R}} k(x-z) \chi_{\left(y+\varepsilon_{i+1}, y+\varepsilon_{i}\right)}(z)\left(b(z)-b_{I}\right) f_{2}(z) \mathrm{d} z\right\}_{i \in \mathbb{N}_{2}, \beta=\left\{\varepsilon_{i}\right\} \in \Theta}\right\|_{F_{\rho}} \\
& +\left\|\left\{\int_{\mathbb{R}} k(x-z) \chi_{\left(x+\varepsilon_{i+1}, x+\varepsilon_{i}\right)}(z)\left(b(z)-b_{I}\right) f_{2}(z) \mathrm{d} z\right\}_{i \in \mathbb{N}_{2}, \beta=\left\{\varepsilon_{i}\right\} \in \Theta}\right\|_{F_{\rho}} \\
= & : L_{3}+L_{4} .
\end{aligned}
$$

By noting that $N_{2}=\left\{i \in Z: \varepsilon_{i}-\varepsilon_{i+1}<y-x\right\}$, we have $\varepsilon_{i}-\varepsilon_{i+1}<y-x \leq 2 h$ with $y \in[x, x+2 h]$. Using Hölder's inequality and (1.3), we have

$$
\begin{aligned}
L_{3} & \lesssim h^{1 / r^{\prime}}\left\|\left\{\left\{\int_{\mathbb{R}} \chi_{\left(y+\varepsilon_{i+1}, y+\varepsilon_{i}\right)}(z) \frac{\left|b(z)-b_{I}\right|^{r}\left|f_{2}(z)\right|^{r}}{|x-z|^{r}} \mathrm{~d} z\right)^{1 / r}\right\}_{i \in \mathbb{N}_{2}, \beta=\left\{\varepsilon_{i}\right\} \in \Theta}\right\|_{F_{\rho}} \\
& =h^{1 / r^{\prime}}\left(\sup _{\beta} \sum_{i \in \mathbb{N}_{1}}\left(\int_{\mathbb{R}} \chi_{\left(y+\varepsilon_{i+1}, y+\varepsilon_{i}\right)}(z) \frac{\left|b(z)-b_{I}\right|^{r}\left|f_{2}(z)\right|^{r}}{|x-z|^{r}} \mathrm{~d} z\right)^{p / r}\right)^{1 / \rho} \\
& \lesssim h^{1 / r^{\prime}}\left(\int_{\mathbb{R}} \frac{\left|b(z)-b_{I}\right|^{r}\left|f_{2}(z)\right|^{r}}{|x-z|^{r}} \mathrm{~d} z\right)^{1 / r} \\
& \lesssim\|b\|_{L i p_{\alpha}} \sum_{k=3}^{\infty} \frac{h^{\alpha}}{2^{k\left(1 / r^{\prime}-\alpha\right)}}\left(\frac{1}{2^{k+1} h} \int_{x}^{x+2^{k+1} h}|f(z)|^{r} \mathrm{~d} z\right)^{1 / r} \\
& \lesssim \sum_{k=3}^{\infty} \frac{\|b\|_{L i p_{\alpha}}}{2^{k / r^{\prime}}}\left(\int_{x}^{x+2^{k+1} h}|f(z) \omega(z)|^{1 / \alpha} \mathrm{d} z\right)^{\alpha}\left(\frac{\|b\|_{L i p_{\alpha}}}{2^{k+1} h} \int_{x}^{x+2^{k+1} h} \omega(z)^{-s} \mathrm{~d} z\right)^{1 / s} \\
& \lesssim\|b\|_{L i p_{\alpha}}\|f \omega\|_{L^{1 / \alpha}} \omega(x)^{-1},
\end{aligned}
$$

where $\omega^{-r}=\omega^{-t /(1-\alpha)} \in A_{1}^{-}$for all $\omega \in A^{+}(1 / \alpha, \infty)$. By similar arguments, we have

$$
L_{4} \lesssim\|b\|_{L i p_{\alpha}}\|f \omega\|_{L^{1 / \alpha}} \omega(x)^{-1},
$$

Following from the estimates of $L_{1}, L_{3}, L_{3}$ and $L_{4}$, we get 


$$
J_{2} \leq J_{21}+J_{22} \leq L_{1}+L_{2}+L_{3}+L_{4} \lesssim\|b\|_{L i p_{\alpha}}\|f \omega\|_{L^{1 / \alpha}} \omega(x)^{-1} .
$$

This together with the estimate of $J_{4}$ implies

$$
\begin{aligned}
& \left\|V\left(\mathcal{T}^{+}\right)\left(\left(b-b_{I}\right) f_{2}\right)(y)-V\left(\mathcal{T}^{+}\right)\left(\left(b-b_{I}\right) f_{2}\right)(x)\right\|_{F_{\rho}} \\
& \leq J_{1}+J_{2} \lesssim\|b\|_{L i i_{\alpha}}\|f \omega\|_{L^{1 / \alpha}} \omega(x)^{-1},
\end{aligned}
$$

where $\omega^{-r}=\omega^{-t /(1-\alpha)} \in A_{1}^{-}$for all $\omega \in A^{+}(1 / \alpha, \infty)$. Then

$$
\left\|\omega M_{3}^{+} f\right\|_{\infty} \lesssim\|b\|_{L i p_{\alpha}}\|f \omega\|_{L^{1 / \alpha}} .
$$

For $1 / r-1 / s=\alpha$ and $A^{+}(p, q)$, it follows from Lemma 2.3 that

$$
\left\|M_{3}^{+} f\right\|_{L^{q}\left(\omega^{q}\right)} \lesssim\|b\|_{L i p_{\alpha}}\|f\|_{L^{p}\left(\omega^{p}\right)} .
$$

This completes the proof.

Now, we turn to the proof of theorem 1.1.

Proof. For $\omega \in A^{+}(p, q)$, by Lemma 2.2, we have $\omega^{q} \in A_{q}^{+}$. Using Lemma 2.4 and Lemma 3.1, we obtain

$$
\begin{aligned}
\left\|\mathcal{V}_{\rho}\left(\mathcal{T}_{b}^{+}\right) f\right\|_{L^{q}\left(\omega^{q}\right)} & \lesssim\left\|M^{+}\left(\mathcal{V}_{\rho}\left(\mathcal{T}_{b}^{+}\right) f\right)\right\|_{L^{q}\left(\omega^{q}\right)} \\
& \lesssim\left\|M^{+, \#}\left(\mathcal{V}_{\rho}\left(\mathcal{T}_{b}^{+}\right) f\right)\right\|_{L^{q}\left(\omega^{q}\right)} \\
& \lesssim\|b\|_{L_{p_{a}}}\|f\|_{L^{p}\left(\omega^{p}\right)} .
\end{aligned}
$$

It remains to prove $\left\|\mathcal{V}_{\rho}\left(\mathcal{T}_{b}^{+}\right) f\right\|_{L^{q}\left(\omega^{q}\right)}<\infty$. By the similar arguments in the proof of Theorem 1.3 in [10], we can get $\left\|\mathcal{V}_{\rho}\left(\mathcal{T}_{b}^{+}\right) f\right\|_{L^{q}\left(\omega^{q}\right)}<\infty$. Then Theorem 1.1 is proved.

\section{Supported}

Supported by the Natural Science Foundation of Shandong Province (No. ZR2020QA006).

\section{Conflicts of Interest}

The authors declare no conflicts of interest regarding the publication of this paper.

\section{References}

[1] Bourgain, J. (1989) Pointwise Ergodic Theorems for Arithmetric Sets. Publications Mathématiques de I Institut des Hautes Études Scientifiques, 69, 5-45. https://doi.org/10.1007/BF02698838

[2] Campbell, J., Jones, R., Reinhdd, K. and Wierdl, M. (2000) Oscillation and Variation for the Hilbert Transform. Duke Mathematical Journal, 105, 59-83. https://doi.org/10.1215/S0012-7094-00-10513-3

[3] Campbell, J., Jones, R., Reinhdd, K. and Wierdl, M. (2003) Oscillation and Variation for Singular Integrals in Higher Dimensions. Transactions of the American 
Mathematical Society, 355, 2115-2137. https://doi.org/10.1090/S0002-9947-02-03189-6

[4] Ding, Y., Hong, G. and Liu, H. (2017) Jump and Variational Inequalities for Rough Operators. Journal of Fourier Analysis and Applications, 23, 679-711.

https://doi.org/10.1007/s00041-016-9484-8

[5] Lépingle, D. (1976) La variation d'ordre $p$ des semi-martingales. Zeitschrift fur Wahrscheinlichkeitstheorie und verwandte Gebiete, 36, 295-316. https://doi.org/10.1007/BF00532696

[6] Le Merdy, C. and Xu, Q. (2012) Strong $q$-Variation Inequalities for Analytic Semigroups. Annales de I Institut Fourier, 62, 2069-2097. https://doi.org/10.5802/aif.2743

[7] Gillespie, T. and Torrea, J. (2004) Dimension Free Estimates for the Oscillation of Riesz Transforms. Israel Journal of Mathematics, 141, 125-144. https://doi.org/10.1007/BF02772215

[8] Ma, T., Torrea, J. and Xu, Q. (2015) Weighted Variation Inequalities for Differential Operators and Singular Integrals. Journal of Functional Analysis, 268, 376-416. https://doi.org/10.1016/j.jfa.2014.10.008

[9] Ma, T. and Xu, Q. (2017) Weighted Variation Inequalities for Differential Operators and Singular Integrals in Higher Dimensions. Science China Mathematics, 60, 1419-1442. https://doi.org/10.1007/s11425-016-9012-7

[10] Liu, F. and Wu, H. (2015) A Criterion on Oscillation and Variation for the Commutators of Singular Integral Operators. Forum Mathematicum, 27, 77-97. https://doi.org/10.1515/forum-2012-0019

[11] Liu, F., Jhang, S., Oh, S. and Fu, Z. (2019) Variation Inequalities for One-Sided Singular Integrals and Related Commutators. Mathematics, 7, 876.

https://doi.org/10.3390/math7100876

[12] Zhang, J. and $\mathrm{Wu}, \mathrm{H}$. (2015) Oscillation and Variation Inequalities for the Commutators of Singular Integrals with Lipschitz Functions. Journal of Inequalities and Applications, 2015, Article No. 214. https://doi.org/10.1186/s13660-015-0737-x

[13] Aimar, H., Forzani, L. and Martín-Reyes, F. (1997) On Weighted Inequalities for One-Sided Singular Integrals. Proceedings of the American Mathematical Society, 125, 2057-2064. https://doi.org/10.1090/S0002-9939-97-03787-8

[14] Sawyer, E. (1986) Weighted Inequalities for the One-Sided Hardy-Littlewood Maximal Functions. Transactions of the American Mathematical Society, 297, 53-61. https://doi.org/10.1090/S0002-9947-1986-0849466-0

[15] Macías, R. and Riveros, M. (2000) One-Sided Extrapolation at Infinity and Singular integrals. Proceedings of the Royal Society of Edinburgh Section A, 130, 1081-1102. https://doi.org/10.1017/S0308210500000585

[16] Martín-Reyes, F., Pick, L. and de la Torre, A. (1993) $A_{\infty}^{+}$Condition. Canadian Journal of Mathematics, 45, 1231-1244. https://doi.org/10.4153/CJM-1993-069-9

[17] Lorente, M. and Riveros, M. (2007) Two Extrapolation Theorems for Related Weighted and Applications. Mathematical Inequalities \& Applications, 10, 643-660. https://doi.org/10.7153/mia-10-60

[18] Martín-Reyes, F. and de la Torre, A. (1994) One-Sided BMO Spaces. Journal of the London Mathematical Society, 49, 529-542. https://doi.org/10.1112/jlms/49.3.529 